\title{
Data assimilation using an ensemble of models: a hierarchical approach
}

\author{
Peter Rayner \\ School of Earth Sciences, University of Melbourne, Melbourne, Australia \\ Correspondence: Peter Rayner (prayner@unimelb.edu.au)
}

Received: 20 January 2017 - Discussion started: 3 February 2017

Revised: 11 July 2019 - Accepted: 25 September 2019 - Published: 27 March 2020

\begin{abstract}
One characteristic of biogeochemical models is uncertainty about their formulation. Data assimilation should take this uncertainty into account. A common approach is to use an ensemble of models. We must assign probabilities not only to the parameters of the models but also to the models themselves. The method of hierarchical modelling allows us to calculate these probabilities. This paper describes the approach, develops the algebra for the most common case and then applies it to the Atmospheric Tracer Transport Model Intercomparison Project (TransCom). We see that the discrimination among models is unrealistically strong, due to optimistic assumptions inherent in the underlying inversion. The weighted ensemble means and variances from the hierarchical approach are quite similar to the conventional values because the best model in the ensemble is also quite close to the ensemble mean. The approach can also be used for crossvalidation in which some data are held back to test estimates obtained with the rest. We demonstrate this with a test of the TransCom inversions holding back the airborne data. We see a slight decrease in the tropical sink and a notably different preferred order of models.
\end{abstract}

\section{Introduction}

Models of any interesting biogeochemical system are inexact. They cannot include all interesting processes, the governing equations of processes are not known exactly or computational resolution limits the accuracy of the solution. Throughout this series we stress that quantitative descriptions should be inherently statistical, meaning they must include information on the probability of any quantity, either inferred or predicted. This requires us to describe the uncertainty in- troduced into any quantity by that of the model. Model uncertainty is of two forms, structural and parametric. Structural uncertainties occur when we do not know the functional forms that relate the inputs and outputs of the real system or that control its evolution. In biogeochemical models these functional forms are exactly specified so that uncertainty is usually manifested as an error. Parametric errors occur when the functional forms are well-known but there is uncertainty in various quantities such as constants in physical equations, initial values or boundary conditions. Uncertainties in model predictions arising from parametric uncertainty can be generated by semi-analytic error propagation (e.g. Scholze et al., 2007; Rayner et al., 2011) or by generating ensembles of model simulations from samples of the probability density functions (PDFs) of parameters (e.g. Murphy et al., 2007; Bodman et al., 2013).

Ensemble methods dominate the study of model uncertainty. The most common approach is model intercomparison of which the Coupled Model Intercomparison Project (Taylor et al., 2012) for the physical climate and $\mathrm{C}^{4} \mathrm{MIP}$ (Friedlingstein et al., 2006) for the global carbon cycle are prominent examples. The MIPs play a crucial but controversial role in quantifying uncertainty. First, they may underestimate uncertainty since it is impossible, even in principle, to know how well a given ensemble properly samples the manifold of possible models. On the other hand, not all models are equally credible. They do more or less well at tests like fitting observations or conserving required quantities. This has led to the application of Bayesian model averaging (e.g. Murphy et al., 2007) in which models are tested against some criteria (such as fit to observations) and their predictions weighted accordingly. 
Inverse problems or data assimilation as discussed in this volume generally treats parametric uncertainty. It uses observations and statistical inference to improve knowledge of the uncertain values (see Rayner et al., 2019, and references therein for a general introduction). Structural model uncertainty must still be included and indeed it often dominates other uncertainties. Model uncertainty is hard to characterise with analytic PDFs since errors in the functional forms will project systematically onto errors in simulated quantities. Hierarchical approaches (e.g. Cressie et al., 2009) provide a mechanism for including uncertainties in the choice of model in the formulation. For an ensemble of models this involves introducing an extra discrete variable (the index of the set of models) into the problem and calculating its probability. This probability goes under several names, e.g. the Bayes factor (Kass and Raftery, 1995) or the evidence (MacKay, 2003, Sect. 28). We can then calculate probability distributions for model parameters as weighted averages over these model probabilities. Hence this application of hierarchical Bayesian modelling is closely related to Bayesian model averaging (Hoeting et al., 1999; Raftery et al., 2005).

Ensemble methods are rare for biogeochemical data assimilation since there are few problems for which a useful population of assimilation systems currently exists. The clearest exception to this is the case of global-scale atmospheric inversions where the Atmospheric Tracer Transport Model Intercomparison Project (TransCom; Gurney et al., 2002, 2003, 2004; Baker et al., 2006) used an ensemble of atmospheric transport models and common inversion systems to infer regional $\mathrm{CO}_{2}$ fluxes from atmospheric concentrations. All these studies faced the problem of estimating properties of the ensemble such as its mean and some measure of spread. Throughout they opted for the ensemble mean and two measures of spread, the standard deviation of the maximum a posteriori (most likely) estimate from each ensemble member and the square root of the mean of the posterior variances of the ensemble. This treated all members of the ensemble equally.

Equal weighting was challenged by Stephens et al. (2007), who compared the seasonality of vertical gradients in model simulations and observations. They found that only a subset of models produced an acceptable simulation and that this subset favoured larger tropical uptake than the ensemble mean. Pickett-Heaps et al. (2011) extended this calculation. They compared simulations using optimised fluxes with airborne profiles. This required simulating the airborne profiles using the optimal fluxes for each model. Of the four atmospheric transport models tested, TM3 (Heimann and Körner, 2003) performed substantially better against these extra data than the other three.

Both the cited studies used data not included in the inversion, a procedure often called cross-validation. Crossvalidation asks whether new data enhance or reduce our confidence in previous estimates while Bayesian model averaging calculates our relative confidence in two models. We shall see that the machinery needed to answer these two questions is very similar.

The outline of the paper is as follows. In Sect. 2 we review the necessary machinery. Section 3 describes an application to the TransCom case. Section 4 describes an extension to treat covarying model errors. Section 5 discusses the use of the machinery for model comparison and cross-validation. Section 6 discusses some computational aspects.

\section{Theory}

The following can be regarded as a development of ideas described in Jaynes and Bretthorst (2003, Sect. 21) or MacKay (2003, Sect. 28).

The standard data assimilation problem seeks to improve knowledge of some target variables in a model given observations. We express our knowledge as probability density functions (PDFs). The true state must be consistent with three independent pieces of information, our prior knowledge of the target variables, our knowledge of the observed quantities, and the relationship between target variables and observations instantiated in an observation operator. In most applications the target variables are continuous quantities such as model parameters or initial or boundary conditions. We form the joint probability by multiplication as

$p\left(\mathbf{x}, \mathbf{y} \mid \mathbf{x}^{\mathrm{b}}, \mathbf{y}^{\mathrm{o}}\right) \propto p\left(\mathbf{x} \mid \mathbf{x}^{\mathrm{b}}\right) \times p\left(\mathbf{y} \mid \mathbf{y}^{\mathrm{o}}\right) \times p(\mathbf{y} \mid H(\mathbf{x}))$,

where $\mathbf{x}$ represents the target variables, $\mathbf{y}$ the true values of the observed quantities and $H$ the observation operator. Usually there is a prior or background value $\mathbf{x}^{\mathrm{b}}$ which serves as a location parameter for $p\left(\mathbf{x} \mid \mathbf{x}^{\mathrm{b}}\right)$. Even more common is a location parameter for $p\left(\mathbf{y} \mid \mathbf{y}^{\mathbf{o}}\right)$, usually an observed value returned by an imperfect measurement system.

We generate the final PDF for $\mathbf{x}$ by integrating over $\mathbf{y}$

$p(\mathbf{x}) \propto \int p(\mathbf{x}, \mathbf{y}) d \mathbf{y}$.

In the usual case of data assimilation we only have one observation operator. Thus we often forget that the posterior PDFs for target variables are implicitly dependent on the observation operator. Where an ensemble of observation operators is available we can no longer assume certainty for which one we should use. The $i$ th observation operator $H_{i}$ becomes part of the target variables so instead of calculating $p(\mathbf{x} \mid \mathbf{y})$ we now seek $p\left(\mathbf{x}, H_{i} \mid \mathbf{y}\right) .{ }^{1}$ Once we have calculated $p\left(\mathbf{x}, H_{i} \mid \mathbf{y}\right)$ we can either integrate over $\mathbf{x}$ if we are interested in the relative probabilities of different observation operators or we can sum over the various choices of observation operators to obtain the PDF for $\mathbf{x}$. The hierarchical approach (see Rayner et al., 2019, Sect. 5.6) factorises this joint PDF of observation

\footnotetext{
${ }^{1}$ The true target variable is $i$, the index variable on the set of observation operators, but we will continue to use $H_{i}$ to make it clear to what this index refers.
} 
operators and continuous target variables using an expression known variously as the chain rule of probabilities or the law of total probabilities. In the case of a discrete choice of observation operator this takes the form

$$
p\left(\mathbf{x}, H_{i}\right)=p\left(\mathbf{x} \mid H_{i}\right) p\left(H_{i}\right) .
$$

We can apply the same rule to the joint probability in Eq. (1) to yield

$p\left(\mathbf{x}, H_{i} \mid \mathbf{y}\right)=P\left(\mathbf{x} \mid \mathbf{y}, H_{i}\right) p\left(H_{i} \mid \mathbf{y}\right)$.

We see that the hierarchical and non-hierarchical PDFs differ only by the factor $p\left(H_{i} \mid \mathbf{y}\right)$ and we hence need to calculate this term.

We will develop the theory for the simplest linear Gaussian case. Here many of the resulting integrals have analytic solutions. The approach will hold for nonlinear observation operators, provided they are approximately linear over enough of the support for the joint distribution of $\mathbf{x}$ and $\mathbf{y}$. The qualitative ranking of models is unlikely to be sensitive to weak nonlinearities since, as we shall see, the discrimination among models is strong.

We follow the notation of Rayner et al. (2019). We switch from using a potentially nonlinear observation operator $H$ to a linear one represented by the Jacobian $\mathbf{H}$. Take a collection of linear observation operators with Jacobians $\mathbf{H}_{1} \ldots \mathbf{H}_{N}$, with prior probability for the continuous target variables given by $G\left(\mathbf{x} \mid \mathbf{x}^{\mathrm{b}}, \mathbf{B}\right)$ and probability for the data given by $G\left(\mathbf{y} \mid \mathbf{y}^{\mathrm{o}}, \mathbf{R}\right)$, where $G(\mathbf{x} \mid \mu, \mathbf{C})$ represents the Gaussian distribution of the variable $\mathbf{x}$ with mean $\mu$ and uncertainty covariance $\mathbf{C}$. $\mathbf{x}^{\mathrm{b}}$ is the mean of the prior distribution for $\mathbf{x}$ (often abbreviated as the background value) and $\mathbf{B}$ is the prior uncertainty covariance of $\mathbf{x}$ (often abbreviated the background or prior uncertainty). $\mathbf{y}^{\mathrm{o}}$ is the mean of the PDF for the observation with $\mathbf{R}$ representing the uncertainty of the observing process. See Rayner et al. (2019) Sect. 5 for a more detailed explanation.

For each $\mathbf{H}_{i}$ our problem is the linear Gaussian inversion described in Rayner et al. (2019) (Sect. 6.4). Most importantly for us the posterior PDF $p\left(\mathbf{x} \mid \mathbf{y}, \mathbf{H}_{i}\right)$ is Gaussian:

$p\left(\mathbf{x} \mid \mathbf{y}, \mathbf{H}_{i}\right)=G\left(\mathbf{x}, \mathbf{x}_{i}^{\mathrm{a}}, \mathbf{A}_{i}\right)$,

where $\mathbf{x}_{i}^{\mathrm{a}}$ is the maximum a posteriori estimate for the $i^{\text {th }} \mathrm{ob}-$ servation operator (often called the analysis) with covariance $\mathbf{A}_{i}$. Substituting Eq. (5) into Eq. (4) we obtain

$p\left(\mathbf{x}, \mathbf{H}_{i} \mid \mathbf{y}\right)=p\left(\mathbf{H}_{i} \mid \mathbf{y}\right) \times G\left(\mathbf{x} \mid \mathbf{x}_{i}^{\mathrm{a}}, \mathbf{A}_{i}\right)$.

Thus $p\left(\mathbf{x}, \mathbf{H}_{i} \mid \mathbf{y}\right)$ is a sum of Gaussian distributions.

$p\left(\mathbf{H}_{i} \mid \mathbf{y}\right)$ is the marginal likelihood for a Gaussian (Michalak et al., 2005, Eq. 10):

$$
\begin{gathered}
p\left(\mathbf{H}_{i} \mid \mathbf{y}\right)=K\left|\mathbf{R}+\mathbf{H}_{i} \mathbf{B} \mathbf{H}_{i}^{T}\right|^{-1 / 2} \exp \left[-\frac{1}{2}\left(\mathbf{y}^{\mathrm{o}}-\mathbf{H}_{i} \mathbf{x}^{\mathrm{b}}\right)^{T}\right. \\
\left.\cdot\left(\mathbf{R}+\mathbf{H}_{i} \mathbf{B} \mathbf{H}_{i}^{T}\right)^{-1} \cdot\left(\mathbf{y}^{\mathrm{o}}-\mathbf{H}_{i} \mathbf{x}^{\mathrm{b}}\right)\right] .
\end{gathered}
$$

Note that $p\left(\mathbf{H}_{i} \mid \mathbf{y}\right)$ is a PDF over the indices $i$ since all terms on the right-hand side of Eq. (7) apart from $\mathbf{H}_{i}$ are fixed. $K$ is chosen so that $\sum_{i} p\left(\mathbf{H}_{i} \mid \mathbf{y}\right)=1$. This normalisation reflects the fact that we must choose one of the models.

\subsection{Interpretation}

One interpretation for the exponential in Eq. (7) is as a ratio of the performance of the model and its expected performance. The term $\mathbf{H}_{i} \mathbf{x}^{\mathrm{b}}-\mathbf{y}^{\mathrm{o}}$ is the mismatch between the observations and the simulation using the background value. Provided $\mathbf{x}$ and $\mathbf{y}$ are independent before our assimilation, $\mathbf{R}+\mathbf{H}_{i} \mathbf{B} \mathbf{H}_{i}^{T}$ is the variance of this prior mismatch. This follows from the Jacobian rule of probabilities (Tarantola, 2005, Eq. 1.18) and the expression for the variance of the difference of two normally distributed quantities. Thus, inspection of the right-hand side of Eq. (7) shows it to be, excluding some potential normalisation, $G\left(\mathbf{z}, 0, \mathbf{H}_{i} \mathbf{B} \mathbf{H}_{i}^{T}+\mathbf{R}\right)$ evaluated at the point $\mathbf{z}=\mathbf{H}_{i} \mathbf{x}^{\mathrm{b}}-\mathbf{y}^{\circ}$. Smaller magnitudes of $\mathbf{H}_{i} \mathbf{x}^{\mathrm{b}}-\mathbf{y}^{\mathrm{o}}$ correspond to better a priori simulations of the observations and higher values of $p\left(\mathbf{H}_{i} \mid \mathbf{y}\right)$, i.e. more likely models. Equal magnitudes of $\mathbf{H}_{i} \mathbf{x}^{\mathrm{b}}-\mathbf{y}^{\mathrm{o}}$ may not produce the same value of $p\left(\mathbf{H}_{i} \mid \mathbf{y}\right)$ since the mismatch variance $\mathbf{H}_{i} \mathbf{B} \mathbf{H}_{i}^{T}+\mathbf{R}$ may not weight them equally. We can say equivalently that the model performance should be judged by the normalised prediction error (simulation - observation divided by its variance) penalised by the expected range of the predictions or the volume of the data space occupied by the prior model and its uncertainty (see discussion in MacKay, 2003, Sect. 28).

Equation (7) occurs in other hierarchical contexts such as the calculation of covariance parameters by Michalak et al. (2005) and Ganesan et al. (2014). This is understandable since the submodels in all three cases are the classical Gaussian problem. We note that these two papers used Eq. (7) to tune covariance parameters, which may change the relative weighting of models. It raises the issue that relative performance of models may depend strongly on whether the inversion is well-tuned for that model. The algorithm in Michalak et al. (2005) consists of tuning a scaling factor for prior covariances to maximise $p\left(\mathbf{H}_{i}\right)$ (though in their case there is only one model). We can test the sensitivity to a uniform scaling of $\mathbf{B}$ and $\mathbf{R}$ by a factor $\alpha$. Increasing $\alpha$ increases the determinant so decreases the first factor of $p\left(\mathbf{H}_{i}\right)$ while it decreases the negative exponent and so increases the second part. The balance is a relatively subtle change. In Sect. 3 we will investigate whether this is enough to change the ranking of models in one example.

Note also that for a given $\mathbf{B}$ and $\mathbf{R}$, Eq. (7) is extremely punishing on inconsistency. For example consider a case with $N$ observations and two models $\mathbf{H}_{1}$ and $\mathbf{H}_{2}$ for which the quantity $\frac{1}{N}\left(\mathbf{y}^{\mathrm{o}}-\mathbf{H} \mathbf{x}^{\mathrm{b}}\right)^{T} \cdot\left(\mathbf{R}+\mathbf{H}_{i} \mathbf{B} \mathbf{H}_{i}^{T}\right)^{-1} \cdot\left(\mathbf{y}^{\mathrm{o}}-\right.$ $\mathbf{H} \mathbf{x}^{\mathrm{b}}$ ) (the mean square mismatch per observation), are 1.0 and 1.01 respectively. With $N=10000$ (by no means unusually large) we see, from substitution into Eq. (7), that $p\left(\mathbf{H}_{1} \mid \mathbf{y}\right) / p\left(\mathbf{H}_{2} \mid \mathbf{y}\right)=e^{50} \approx 10^{22}$. This is unrealistic and is an 
example of the "curse of dimensionality" (Stordal et al., 2011) in which distances between points in high-dimensional spaces tend to infinity. We shall address one approach to resolving this problem in Sect. 4.

\subsection{Relationship with other criteria}

The exponent in Eq. (7) is also the minimum value of the cost function usually minimised to solve such systems. It is often denoted $1 / 2 \chi^{2}$. In an assimilation where variances of prior PDFs and residuals calculated from the assimilation are consistent, the expected value of $\chi^{2}$ is equal to the number of observations (Tarantola, 1987, p. 211). We often quote the normalised $\chi^{2}$ as $\frac{\chi^{2}}{n}$, roughly the mean square mismatch per observation.

$p\left(\mathbf{H}_{i} \mid \mathbf{y}\right)$ is related to several other measures of model quality. For convenient comparison we define

$L=-2 \log \left(\frac{p\left(\mathbf{H}_{i} \mid \mathbf{y}\right)}{K}\right)=\log \left|\mathbf{H}_{i} \mathbf{B H}_{i}^{T}+\mathbf{R}\right|+\chi^{2}$.

The change of sign means smaller values of $L$ correspond to more likely models.

$L$ is related to other criteria for model selection such as the Akaike information criterion (AIC; Akaike, 1974) and Schwartz information criterion (also called the Bayesian information criterion, BIC) (Schwarz, 1978). In our case the AIC can be defined as

$\mathrm{AIC}=2 M+\chi^{2}$,

where $M$ is the number of target variables (the dimension of $\mathbf{x})$. The related Bayesian or Schwartz information criterion is defined as

$\mathrm{BIC}=\chi^{2}+M \ln (N)$.

All three criteria consider the goodness of fit of the model. All criteria penalise models for adding parameters. Neither AIC nor BIC take account of different prior uncertainties among parameters or different sensitivities of the observations to these parameters.

\section{The TransCom example}

The TransCom III intercomparison (Gurney et al., 2002, 2004; Baker et al., 2006) was designed to investigate the impact of uncertainty in atmospheric transport models on the determination of $\mathrm{CO}_{2}$ sources and sinks. The target variables were the mean $\mathrm{CO}_{2}$ flux from each of 22 regions (11 land and 11 ocean) for the period 1992-1996. These fluxes excluded fossil fuel emissions and a data-driven estimate based on ocean and atmosphere measurements (Takahashi et al., 1999). Prior estimates and uncertainties were gathered from consultation with experts in each domain. The data were the average $\mathrm{CO}_{2}$ concentration from 76 stations, and the same data were used in every inversion. Participants in the intercomparison calculated Jacobians by inserting a unit flux into an atmospheric transport model corresponding to each region. There were 17 participating models so our space of target variables consists of 22 flux components and an indexed set of 17 models $\mathbf{H}_{i}$.

The inversions for the flux components are carried out by changing $\mathbf{H}$ with all other aspects held constant. The authors then created pooled estimates of the posterior fluxes such as the mean, the mean uncertainty (averaging all the posterior uncertainties) and finally the "between-model" spread, calculated as the covariance among the posterior fluxes for each model. In all these calculations we weighted every model equally. What happens if we apply the methods described in Sect. 2 to calculate pooled estimates?

Figure 1 shows a slightly modified $L$ for the 17 models for the cases without (panel a) and with (panel b) tuning following Michalak et al. (2005). The modification consists of displaying $\log _{10}$ rather than the natural logarithm. For the tuning cases we used one multiplier each for $\mathbf{B}$ and $\mathbf{R}$. We see a large range of weights, 11 orders of magnitude for the untuned cases and 14 orders of magnitude for the tuned cases. This certainly reflects the curse of dimensionality mentioned earlier. For the same reason there is a strong focus of weight on a few models. Tuning intensifies this focus though it leaves the ranking almost unchanged. We conclude therefore that variation in model performance (as measured by $L$ ) does not reflect the quality of tuning of the inversion but something more fundamental about the models and data. Henceforth we consider only the untuned case.

Although rankings do not change much, we see that model 3 and, to a lesser extent, model 1 have much lower weights after tuning than before. The variance tuning procedure reduces the variances for most models (indicating that they fit the data better than the original variances suggest they should). All else being equal, a lower optimal value for the variance scaling factors means an increased $p\left(\mathbf{H}_{i} \mid \mathbf{y}\right)$. Models 1 and 3 do not have their variance scaling changed much so their relative weight is reduced. The reduction is large because of the same dimensionality arguments made above.

In the next two sections we consider the marginal probabilities to investigate the relative probabilities of different models and the pooled flux estimates.

\subsection{Model probabilities: comparing model performance}

The Gaussian weights derived in Sect. 2 are the probabilities that a given model is the correct one for matching the data under the assumption that we must choose one (see Jaynes and Bretthorst, 2003, p. 136 for a discussion of this point). We must, however, be careful not to over-interpret these probabilities as measures of model quality. In the first place, $L$, like the BIC and $\chi^{2}$, grows with the number of observations. The divergence among models then also grows, an effect inten- 


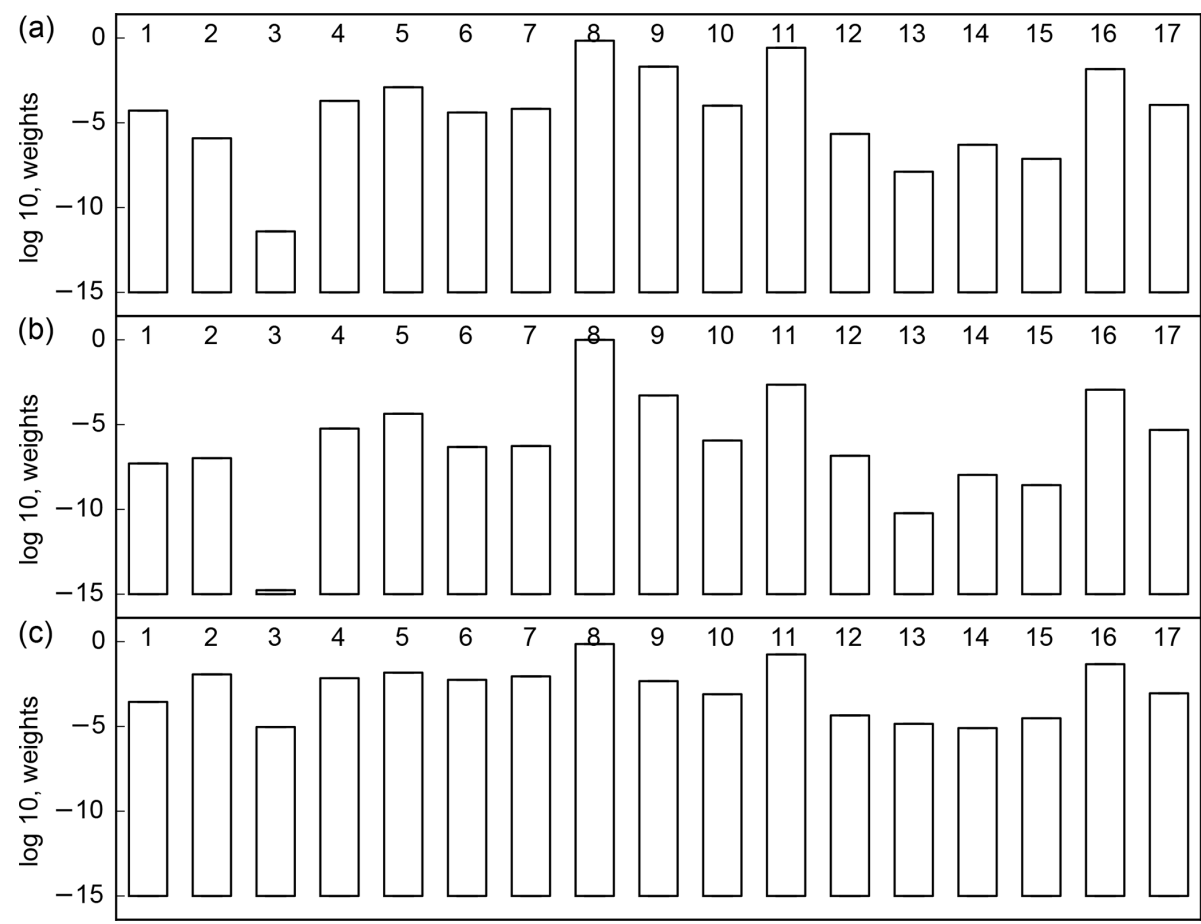

Figure 1. The $\log _{10}$ of $p\left(\mathbf{H}_{i} \mid \mathbf{y}\right)$ for the untuned (a) and tuned (b) cases with residuals used for $\mathbf{R}$ (c) TransCom inversions.

sified when we take exponentials to calculate probabilities. The relative quality of two models depends on the amount of data used to compare them even if our ability to distinguish between them does increase as we add data. We can normalise by considering $L / N$ (where $N$ is the number of observations) as a generalisation of the normalised $\chi^{2}$. This ranges from a minimum of 0.01 to 0.67 . The very low value should not be interpreted as representing an absolute quality of fit since we have normalised the probabilities to sum to 1. Rather it tells us that the apparently large change in the weights is a result of much smaller differences in the relative quality of the fit coupled to large amounts of data.

\subsection{Ensemble means and variances}

Once we sum over $i$ we obtain $p(\mathbf{x} \mid \mathbf{y})$ as a sum of Gaussian distributions with fixed weights. These are usually referred to as Gaussian mixture distributions. We can calculate various statistics of the ensemble using well-known properties of Gaussian mixtures. The mean is calculated as

$\mu=\sum_{i} p\left(\mathbf{H}_{i} \mid \mathbf{y}\right) \mathbf{x}_{i}^{\mathrm{a}}$.

Note that this collapses to the conventional mean if all weights are equal. The variance is calculated as

$\mathbf{A}^{*}=\sum_{i} p\left(\mathbf{H}_{i} \mid \mathbf{y}\right)\left[\mathbf{A}_{i}^{*}+\left(\mathbf{x}_{i}^{\mathrm{a}}-\mu\right)^{2}\right]$.

The superscript $*$ indicates we consider only the diagonal of the relevant matrices; Eq. (12) only accounts for the vari- ance not the covariance of the estimates. The second term in Eq. (12) includes the spread of the means for each model. If all the $p\left(\mathbf{H}_{i} \mid \mathbf{y}\right)$ values are equal, Eq. (12) collapses to the "total uncertainty" metric used by Rayner (2004) to incorporate both the within- and between-model uncertainty described in Gurney et al. (2002).

Figure 2 shows the equally weighted and probabilityweighted cases for the TransCom regions, in a format following Gurney et al. (2002). Here we do not show the within- and between-model metrics separately since the Gaussian mixture naturally combines them. The focus of $p\left(\mathbf{H}_{i} \mid \mathbf{y}\right)$ on a few models ( $70 \%$ on one model) might suggest that the uncertainty in the weighted case should be far smaller than the equally weighted traditional case. Figure 2 shows this is not the case. Both the means and uncertainties for the two cases are quite similar.

The agreement of the means is explained by a result from Gurney et al. (2002). They noted that the mean simulation from their equally weighted ensemble produces a better match to the data than any individual model. The probabilityweighted flux is constructed to maximise the posterior probability across the model ensemble and parameter PDFs; thus its mean should also produce a good match. It is hence no surprise that the preferred model 8 is the model closest to the unweighted model mean. Recalling that the ensemble weights this preferred model at $70 \%$, we see good agreement between weighted and unweighted means.

The similarity in the weighted and unweighted total uncertainty is partly a result of the weak data constraint in 


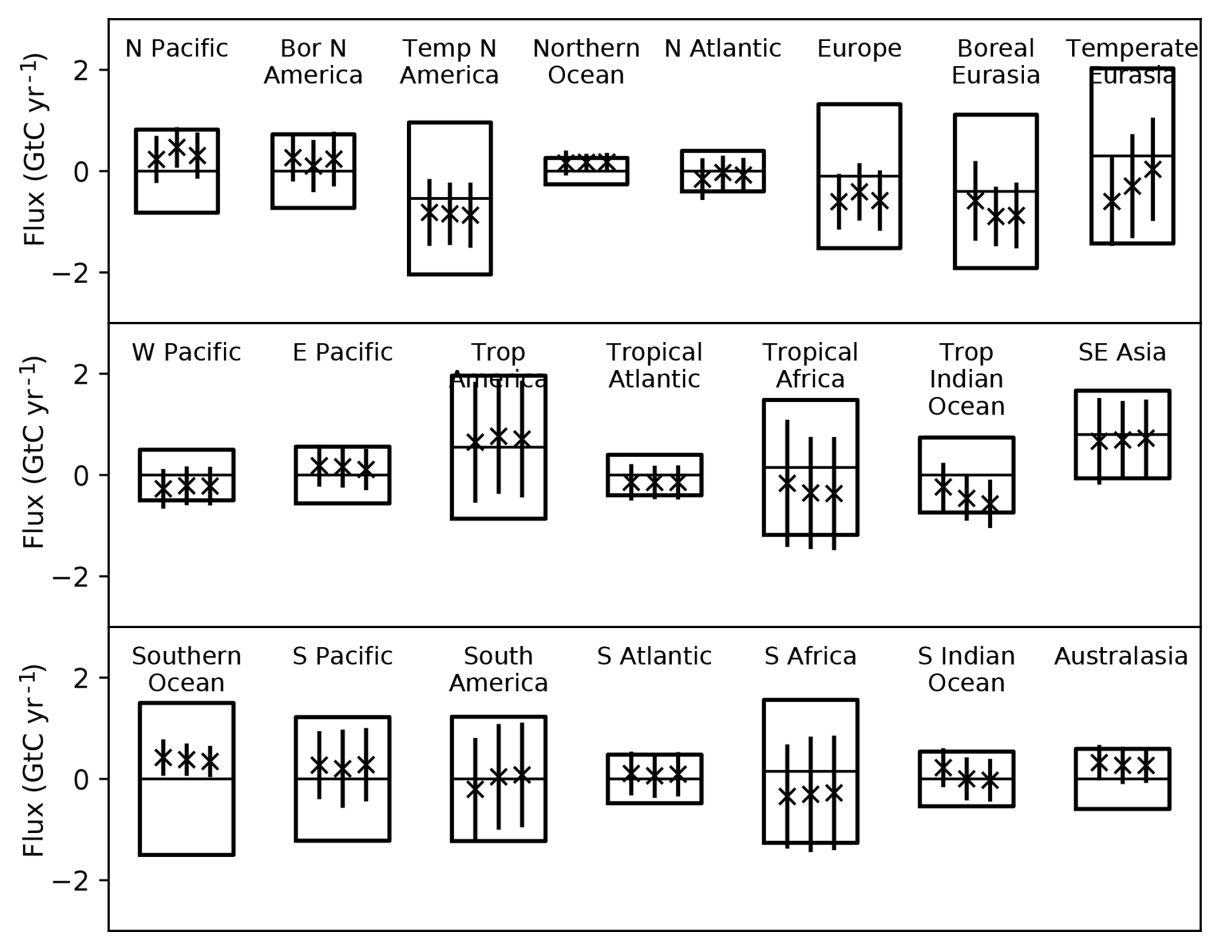

Figure 2. Prior and posterior uncertainties for regional fluxes from TransCom following Gurney et al. (2002). The centre line of each box shows the prior estimate of the mean while the box limits show the $\pm 1 \sigma$ uncertainties. The three bars show the mean (marked with "x") and $\pm 1 \sigma$ uncertainty denoted by the length of the bar. The uncertainty is that of the ensemble including both the uncertainty for each model and the dispersion among model means. The left bar shows the equally weighted case, the middle bar the case for the $p\left(\mathbf{H}_{i} \mid \mathbf{y}\right)$ and the right bar the case with covariance of residuals included.

our problem. Gurney et al. (2002) noted that for almost all regions the within-model uncertainty was larger than the between-model uncertainty. Furthermore the posterior uncertainties produced by each model are rather similar so that the weighted and unweighted contributions in Eq. (12) are similar. The contributions of the between-model uncertainty are different in the weighted and unweighted cases, but, since these are smaller than the other contribution, we do not see a large final difference. This would change in cases where the constraint afforded by the data (as evidenced by the uncertainty reduction compared to the prior) was large.

\section{Improved treatment of observational covariance}

Although mathematically correct, the strong discrimination among models by $L$ is not intuitively reasonable. One reason for the strength of the discrimination is that each data point makes an independent contribution to the PDF. This is not an error in the formulation of $L$ but rather the PDF associated with the data in the underlying assimilation. ${ }^{2}$ In the case of atmospheric transport models this assumption says that if

\footnotetext{
${ }^{2}$ Strictly speaking it is the model PDF from Rayner et al. (2019), but we have combined model and data uncertainties following their Sect. 6.4 .
}

a model makes an error at one station, one cannot assume it will make a similar error at a nearby station. The physical coherence of atmospheric transport processes makes this most unlikely, even if subgrid heterogeneity lends some independence to the two stations.

There are two major approaches to characterising the model error covariance, either a priori or a posteriori. A priori we would like some machinery for calculating how uncertainties in model components or drivers project into model simulations. Lauvaux et al. (2009), for example, described a mechanism for calculating correlations in simulated tracer distributions due to correlated meteorological uncertainty but this is not a comprehensive description, i.e. it leaves out many sources of uncertainty. If we have an ensemble of models we can use the ensemble of simulations using the prior value of the target variables as a measure of the model contribution to uncertainty. This was suggested by Tarantola (1987). The motivating argument is that the ensemble of models samples the uncertainty of the observation operator while maintaining physical consistency for each member of the ensemble. Equation (1) requires the PDF of the simulation $H(\mathbf{x})$ for any x. Tarantola (1987) suggests that the covariance of this PDF can be calculated using $\mathbf{x}^{\mathrm{b}}$.

First define the model mean

$\mu^{\mathrm{b}}=\overline{\mathbf{H} \mathbf{x}^{\mathrm{b}}}$. 
where the average is taken over the ensemble of models. We can then write the ensemble covariance as

$\mathbf{R}_{i, j}^{\text {prior }}=\overline{\left(\mathbf{H} \mathbf{x}_{i}^{\mathrm{b}}-\mu_{i}^{\mathrm{b}}\right)\left(\mathbf{H} \mathbf{x}_{j}^{\mathrm{b}}-\mu_{j}^{\mathrm{b}}\right)^{\mathrm{T}}}$,

where once again the average is over the ensemble of models and the subscripts index the observations.

The second approach is analysis of the posterior residuals. Desroziers et al. (2005) noted that the residuals must be consistent with the PDF assumed for the model-data mismatch, here described by $\mathbf{R}$. If this is not the case we need to make a correction to $\mathbf{R}$. Here again we have a range of choices. If we have enough data we can fit covariance models as functions of space and time. We do not have enough data so we directly calculate the ensemble covariance of the residuals as

$\mathbf{R}_{i, j}^{\text {sample }}=\overline{\left(\mathbf{H} \mathbf{x}_{i}^{\mathrm{a}}-\mathbf{y}_{i}\right)\left(\mathbf{H} \mathbf{x}_{j}-\mathbf{y}_{j}\right)^{\mathrm{T}}}$,

where the overbar denotes an average over the ensemble of models and their respective analyses, and the indices $i$ and $j$ refer to observations. Descriptively $\mathbf{R}^{\text {sample }}$ will be positive if, on average, models make errors of the same sign for observations $i$ and $j$. Note that if the ensemble of models is smaller than the number of observations (usually the case) then both $\mathbf{R}^{\text {sample }}$ and $\mathbf{R}^{\text {prior }}$ are singular. Neither $\mathbf{R}^{\text {prior }}$ nor $\mathbf{R}^{\text {sample }}$ capture observational error however. Tarantola (2005, Eq. 1.106) points out that, for Gaussian PDFs, we can combine the PDFs for the model and observations by adding their respective covariances.

We note in advance an objection to using $\mathbf{R}^{\text {sample }}$ that, by using the residuals, we are double-counting information in any subsequent inversion. This is partly true, although firstly we only use it to correct the spread not the location of the related PDFs, and secondly the same objection holds for any use of posterior diagnostics.

Figure 3 shows, for a sample of stations, the first-guess and residual standard deviations from Eqs. (14) and (15) as well as the control standard deviation. The standard deviations show somewhat similar structure, with the largest values for terrestrially influenced stations such as the Baltic Sea (bal), Hungary(hun) and the Tae-ahn Peninsula, South Korea (tap). Magnitudes of the first-guess variances are larger however. There are 49 of the 76 stations where the first-guess variance is larger than the observational error variance but only one station (Mould Bay in Canada) where this is true for the residual variance. This reflects the convergence of simulations towards the observations. Covariances among stations are more complex, but, as expected, they are strong whenever multiple measurements occur near each other (e.g. at one station or in one vertical profile). This has the desirable property of de-weighting these measurements relative to an independent observational covariance.

The weights for the case using $\mathbf{R}^{\text {sample }}$ are shown as Fig. 1c, and the impact on regional estimates is shown as the right bar in Fig. 2. The ranking is similar to the other cases, especially for the preferred models. The main effect of including the residual covariance is to reduce the penalty for the least-preferred models. Given the small changes among the preferred models it is no surprise that there is little change in the regional estimates or total uncertainties. One reason for the largest impact falling on the least preferred models is that the residual covariance is dominated by the largest residuals, which come from the least preferred models.

\section{Model comparison and cross-validation}

In Sect. 3 we applied the theory to the simplest possible case of models with identical dimensionality and uncertainties; they differed only in their Green's function. The theory is more general than this. We noted in Sect. 2.1 that model performance is determined by the normalised prediction error and the volume of the data space occupied by the prior model. Neither of these depends directly on the dimensionality of the prior model. We can compare a model with two highly uncertain parameters against another with four more certain parameters. This extends the BIC which considers only the number of parameters. The case is quite common in biogeochemistry in which we often compare simple models with empirical and highly uncertain parameters with complex, physically based models whose parameters can be linked to field experiments.

A special case occurs when we compare the prior and posterior models. This is usually done by holding back a subset of the data and testing the improvement in the fit to those data (e.g. Peylin et al., 2016). The approach is frequently called cross-validation. $L$ provides a good basis for comparison of the prior and posterior models. Most importantly it accounts for the different volumes in the data space occupied by the prior and posterior models. Posterior models (informed by the previous assimilation) always occupy less volume in the space of the cross-validation data than their unconstrained or free-running prior model. Thus a good fit to the crossvalidation data is less likely to be a chance event.

It is also possible to weight model estimates by their ability to fit cross-validation data. The steps are as follows:

1. divide data into assimilation and validation data;

2. carry out an ensemble of assimilations using each model and the assimilation data;

3. calculate $L$ using the posterior estimates from step two and the validation data;

4. calculate ensemble statistics from the posterior estimates from step two and $L$ from step three.

Note that the prior means and covariances in Eq. (7) for step three are the posterior means and covariances from step two. Thus, while in Sect. 3.1 we varied only the model $\mathbf{H}$ 

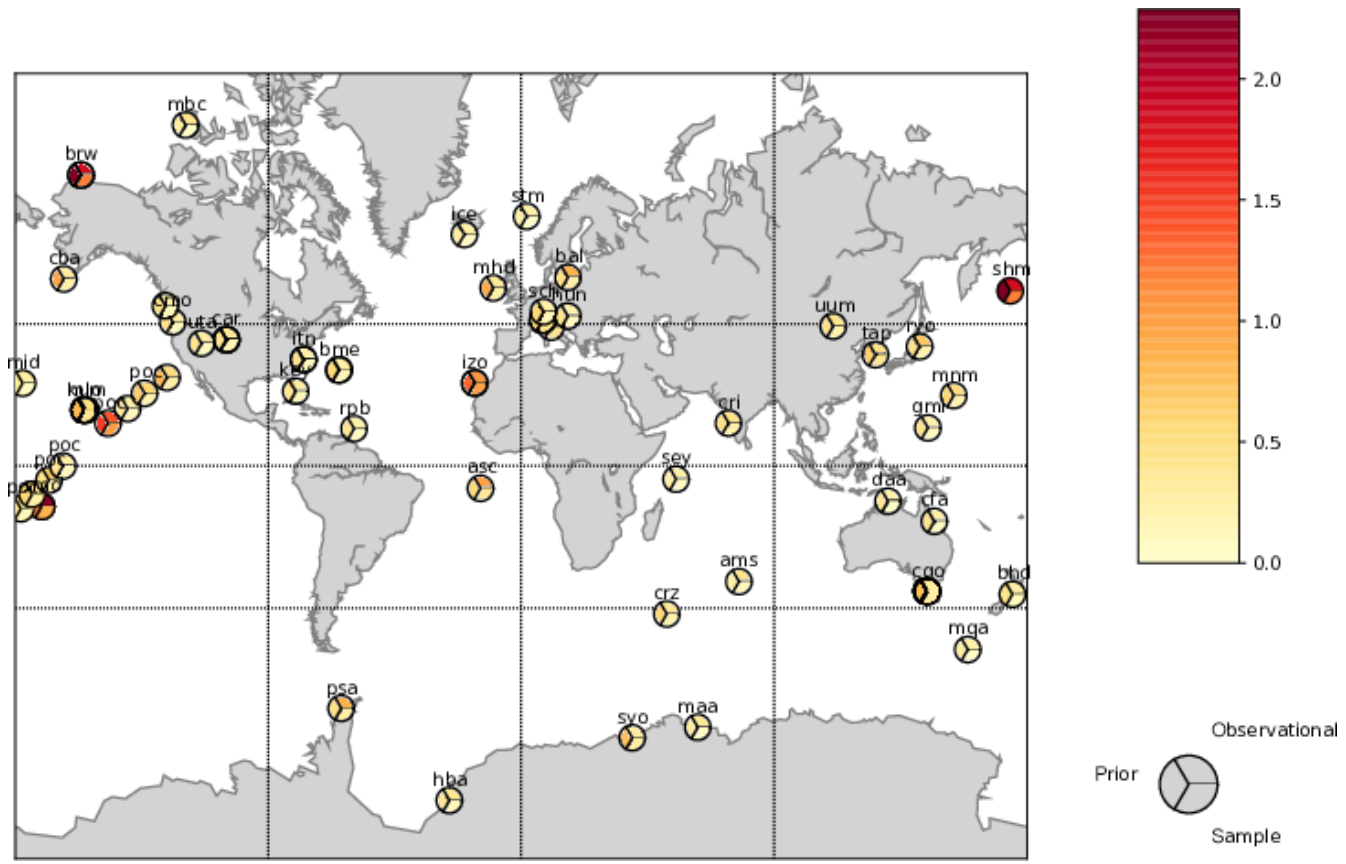

Figure 3. Assumed standard deviations, standard deviations taken from the diagonal of $\mathbf{R}^{\text {prior }}$ and $\mathbf{R}^{\text {sample }}$ for a representative subset of stations.

here we also vary $x^{\mathrm{b}}$ and $\mathbf{B}$. Variations in $\mathbf{B}$ or, more generally, variations in the projection of prior uncertainty into observation space are not usually treated in cross-validation studies (e.g. Pickett-Heaps et al., 2011).

For our example we parallel the test of Stephens et al. (2007). They held back data from airborne profiles and rated models according to their ability to fit seasonal changes in vertical gradients. We cannot use the same measure in our annual mean experiment but we do use the nine points from the airborne profiles above Cape Grim, Tasmania, or Colorado, USA.

We can calculate $L$ using these nine measurements and the prior and posterior models. The comparison of $L$ for these cases shows whether the fit to the data held back from the inversion has improved. One would hope so but Peylin et al. (2016) showed that this is not always the case. In our case $L$ improves by several orders of magnitude due to both a reduction in the residuals and a narrowing of the PDF. Figure 4 shows the comparison of normalised $L$ for the prior (panel a) and posterior (panel b) models. The prior case shows little variation around the equally weighted value of $1 / 17$, while this variation is considerably increased for the posterior case. Figure 5 shows the ensemble statistics for three inversion cases. The left bar is the equally weighted case for the entire network (the left bar from Fig. 2), the middle bar shows the equally weighted case for the inversion with the nine crossvalidation stations removed while the right bar shows the same inversion but weighted according to $p\left(\mathbf{H}_{i} \mid \mathbf{y}^{\mathrm{cv}}\right)$, where $\mathbf{y}^{\mathrm{cv}}$ is the cross-validation data. Averaged across all regions the impact of changing network and changing weighting are comparable, although the largest changes are in North America and South America following from the change of network. This was also observed by Pickett-Heaps et al. (2011).

\section{Computational aspects}

The hardest part of the calculation of $p\left(\mathbf{H}_{i} \mid \mathbf{y}\right)$ is calculating the matrix $\mathbf{H}_{i} \mathbf{B} \mathbf{H}_{i}^{T}+\mathbf{R}$. There are several possible routes depending on the size of the problem and the available machinery. In problems with few parameters it may be possible to calculate and store $\mathbf{H}_{i}$ directly. Recall that $\mathbf{H}_{i}=\nabla_{\mathbf{x}} \mathbf{y}$. We can calculate $\mathbf{H}_{i}$ either as the tangent linear of $H$ (Griewank, 2000) or via finite difference calculations in which a parameter is perturbed. Once we calculate $\mathbf{H}$ we can generate the eigenvalues of $\mathbf{H}_{i} \mathbf{B} \mathbf{H}_{i}^{T}+\mathbf{R}$ from the singular values of $\mathbf{H}_{i}$. In other cases $\mathbf{R}$ is sparse, in which case we can calculate $\left(\mathbf{H}_{i} \mathbf{B} \mathbf{H}_{i}^{T}+\mathbf{R}\right)^{-1}$ as a correction to $\mathbf{R}^{-1}$ using the Sherman-Morrison-Woodbury formula (Cressie and Johannesson, 2008).

If the problem is too large or the generation of the Jacobian too costly, we need to generate an approximation of the determinant of $\mathbf{H}_{i} \mathbf{B H}_{i}^{T}+\mathbf{R}$. A common approach is to calculate the leading eigenvalues of (the symmetric matrix $\mathbf{H}_{i} \mathbf{B H}_{i}^{T}$ ) through a so-called matrix-free approach. Rather than an explicit representation of the matrix, matrix-free approaches require the capability to evaluate the product of the matrix in question with any given vector. The prime example of a matrix-free approach was published by Lanczos 


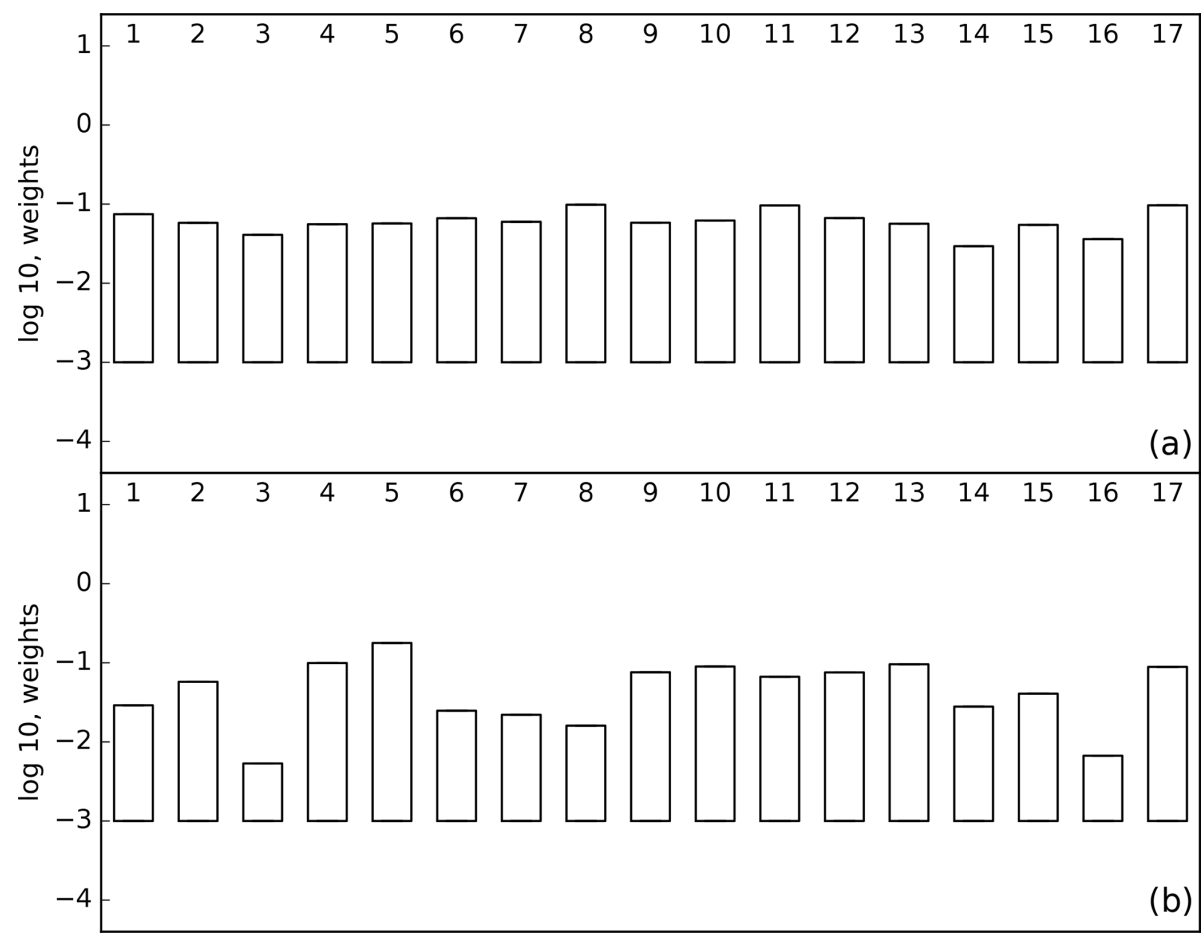

Figure 4. The $\log _{10}$ of $p\left(\mathbf{H}_{i} \mid \mathbf{y}\right)$ for the prior (a) and posterior (b) with $p\left(\mathbf{H}_{i} \mid \mathbf{y}\right)$ calculated using nine airborne measurements over Cape Grim and Colorado.

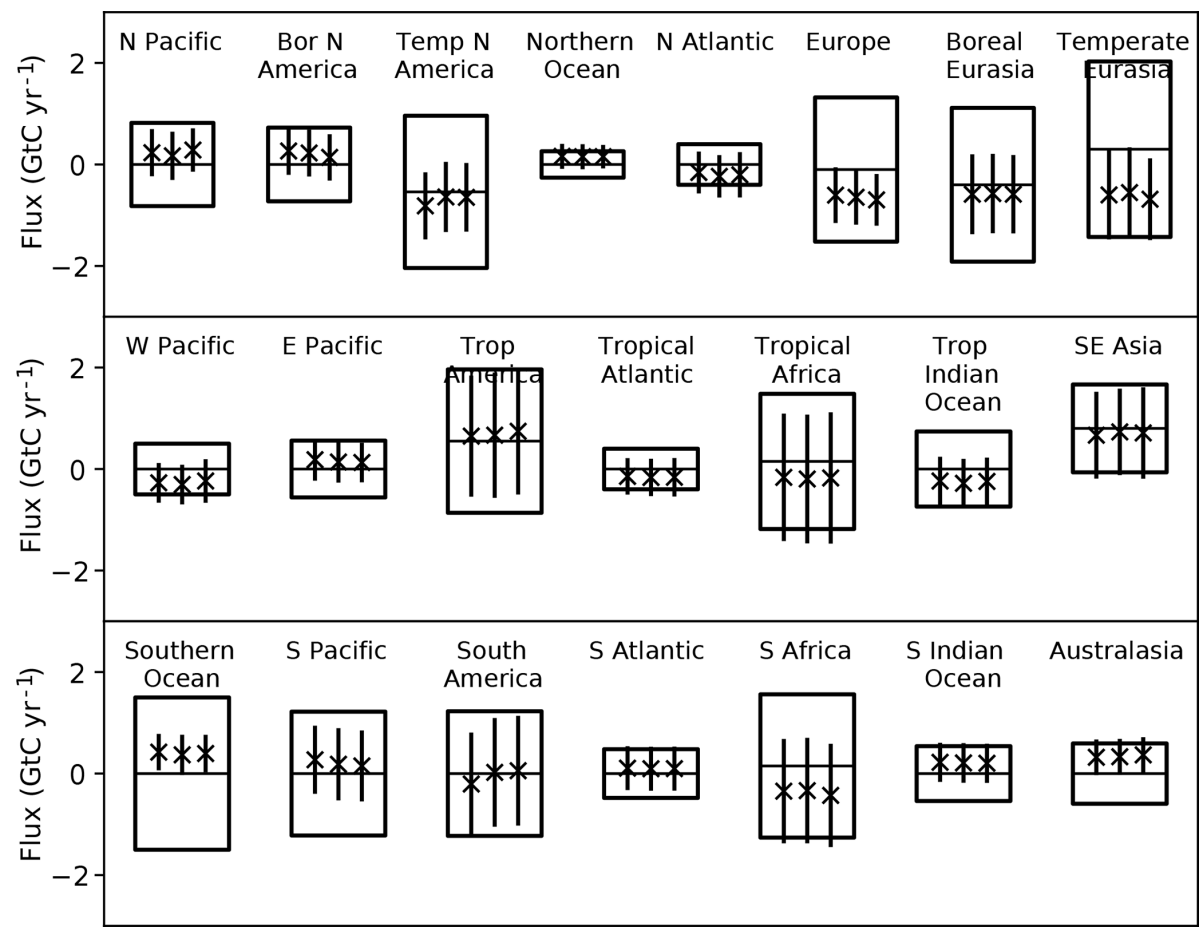

Figure 5. Prior and posterior uncertainties for regional fluxes from TransCom following Gurney et al. (2002). The centre line of each box shows the prior estimate of the mean while the box limits show the $\pm 1 \sigma$ uncertainties. The three bars show the mean (marked with "x") and $\pm 1 \sigma$ uncertainty denoted by the length of the bar. The uncertainty is that of the ensemble including both the uncertainty for each model and the dispersion among model means. The left bar shows the equally weighted case for the full network, the middle bar the equally weighted case with the cross-validation stations removed and the right bar the $L$-weighted case for the cross-validation data. 
(1950). In our case the application of a matrix-free approach requires the tangent linear of $H_{i}$ to generate $\mathbf{H}_{i} \mathbf{x}$ and the adjoint model to generate $\mathbf{H}_{i}^{\mathbf{t}} \mathbf{x}$. This is similar to calculations performed in the conjugate gradient algorithm for the assimilation problem itself (Fisher, 1998). The second term in Eq. (7) is the Bayesian least-squares cost function evaluated at the minimum, so, provided we want to calculate $x^{\mathrm{a}}$ and not just $p\left(\mathbf{H}_{i} \mid \mathbf{y}\right)$, we already have this value.

\section{Discussion and future work}

The method we have outlined points out one way of incorporating measures of model quality into ensemble estimates. The TransCom case points out its main limitation, a strong dependence on the underlying PDFs. The same limitation holds for other calculations with the underlying PDFs, especially measures of information content or posterior uncertainty. Thus the largest effort needed to improve our calculation is the same as that for many other aspects of assimilation, namely the assessment of the independent information available from large sets of observations, accounting for systematic errors in observation operators. This problem is particularly difficult in biogeochemical assimilation. The normal application is of a single assimilation carried out over the longest possible period. This is desirable because there are usually few data available in any period (encouraging maximising the assimilation window) and many of the processes we seek to elucidate are slow so that long windows are desirable to reveal them. This means that it is hard to separate systematic errors arising from the prior, the data themselves or the observation operator.

Some assimilation problems are less subject to this weakness. In numerical weather prediction, for example, we have repeat assimilations. Thus we can test that the underlying PDFs are consistent with their realisations. We also have more direct tests of the quality of the assimilation via forecast skill. The above argument suggests a strong need for ensemble approaches in biogeochemical assimilation.

A more immediate application than properly weighting an ensemble of models may be in model development. Here a common question is of complexity over simplicity. If, as is argued throughout this series, assimilation is a good guide to parameter choice and even structure in models, we need some way to tell whether adding extra processes, with their concomitant uncertainties, is worth the effort. This is a standard problem in statistical inference. The Bayesian formulation outlined here shifts the comparison of two models from complexity to the volume of data space available to them, allowing both complexity and uncertainty to play a role. This offers a promising basis for comparing different versions of a model.

The comparison between models and data sets is, however, incomplete. We cannot easily compare two assimila- tions with different amounts of data since $p(\mathbf{H} \mid \mathbf{y})$ has a strong dependence on dimension.

\section{Conclusions}

We have developed a simple application of hierarchical data assimilation to incorporate choice among an ensemble of models. We have demonstrated it for a computationally simple case, the annual mean version of TransCom. The method provides unrealistically strong discrimination among models, mainly due to incorrect assumptions about underlying PDFs. We have also successfully applied the technique to the crossvalidation of the TransCom inversions by holding back airborne data over Tasmania and Colorado. The method, when coupled with more sophisticated diagnostics of model-data mismatch, should prove a useful extension to traditional biogeochemical data assimilation.

Code and data availability. The code and data files to run the TransCom example and generate the figures in the paper can be found at https://figshare.com/articles/Code_needed_to_run_ the_transcom_ensemble_weighted_probability_case_for_Data_ Assimilation_using_an_Ensemble_of_Models_A_hierarchical_ approach_Geoscience_Model_Development_Discussions_2016_ w_draft_item/4210212 (last access: 5 March 2020; Rayner, 2016). 
We proceed via the multiplication of PDFs described in Rayner et al. (2019, Sect. 4). We start with a uniform prior distribution for the choice of our $N$ models $K\left(\mathbf{H}_{i}\right)=\frac{1}{N}$ and Gaussian PDFs for prior estimates of parameters and for data. Our problem consists of finding the marginal probability $p\left(\mathbf{H}=\mathbf{H}_{i}\right)$.

Using Eq. (1.93) from Tarantola (2005) we have

$p\left(\mathbf{H}_{i}, \mathbf{x}\right)=\frac{K\left(\mathbf{H}_{i}\right) G\left(\mathbf{x}, \mathbf{x}^{\mathrm{b}}, \mathbf{P}\right) \cdot G\left(\mathbf{H}_{i} \mathbf{x}, \mathbf{y}, \mathbf{R}\right)}{\sum_{i} \int K\left(\mathbf{H}_{i}\right) G\left(\mathbf{x}, \mathbf{x}^{\mathrm{b}}, \mathbf{P}\right) G\left(\mathbf{H}_{i} \mathbf{x}, \mathbf{y}, \mathbf{R}\right) \mathrm{d} x}$.

We wish to find

$p\left(\mathbf{H}=\mathbf{H}_{i}\right)=\int \sigma\left(\mathbf{H}_{i}, \mathbf{x}\right) \mathrm{d} x$

and also

$p(\mathbf{x})=\sum_{i} p\left(\mathbf{x}, \mathbf{H}_{i}\right)$.

\section{Model probability}

The denominator is a normalisation, so if we worry only about relative likelihoods we need only the integral of the numerator. The multivariate Gaussian can be expanded as

$$
\begin{aligned}
& G(\mathbf{x} \mu, \mathbf{C})=(2 \pi)^{-n / 2}|\mathbf{C}|^{-1 / 2} \\
& \quad \exp \left[-\frac{1}{2}(\mathbf{x}-\mu)^{T} \cdot \mathbf{C}^{-1} \cdot(\mathbf{x}-\mu)\right],
\end{aligned}
$$

where $n$ is the dimension of $\mu$.

Substituting Eq. (A4) into Eq. (A1) gives

$$
\begin{aligned}
& p\left(\mathbf{H}_{i}, \mathbf{x}\right) \propto|\mathbf{P}|^{-1 / 2}|\mathbf{R}|^{-1 / 2} \\
& \exp \left[-\frac{1}{2}\left(\mathbf{x}-\mathbf{x}^{\mathrm{b}}\right)^{T} \cdot \mathbf{P}^{-1} \cdot\left(\mathbf{x}-\mathbf{x}^{\mathrm{b}}\right)\right] \\
& \exp \left[-\frac{1}{2}(\mathbf{H} \mathbf{x}-\mathbf{y})^{T} \cdot \mathbf{R}^{-1} \cdot(\mathbf{H} \mathbf{x}-\mathbf{y})\right] .
\end{aligned}
$$

To simplify this expression we note that determinant is distributive over multiplication and also that multiplying exponentials is achieved by adding exponents. With some linear algebra, completing the square and the use of the special form of the matrix inversion lemma

$\left(\mathbf{P}^{-1}+\mathbf{H}^{T} \mathbf{R}^{-1} \mathbf{H}\right)^{-1}=\mathbf{P}-\mathbf{P} \mathbf{H}^{T}\left(\mathbf{H} \mathbf{P} \mathbf{H}^{T}+\mathbf{R}\right)^{-1} \mathbf{H P}$,

we can reduce Eq. (A5) to the form

$$
\begin{aligned}
& p\left(\mathbf{H}_{i}, \mathbf{x}\right) \propto|\mathbf{P}|^{-1 / 2}|\mathbf{R}|^{-1 / 2} \\
& \exp \left[-\frac{1}{2}(\mathbf{x}-\mu)^{T} \cdot \mathbf{A}^{-1} \cdot(\mathbf{x}-\mu)\right] \\
& \quad \times \exp \left[-\frac{1}{2}\left(\mathbf{y}-\mathbf{H}_{i} \mathbf{x}^{\mathrm{b}}\right)^{T} \cdot\left(\mathbf{R}+\mathbf{H}_{i} \mathbf{P} \mathbf{H}_{i}^{T}\right)^{-1} \cdot\left(\mathbf{y}-\mathbf{H}_{i} \mathbf{x}^{\mathrm{b}}\right)\right],
\end{aligned}
$$

where

$\mu=\mathbf{x}^{\mathrm{b}}+\mathbf{P} \mathbf{H}_{i}^{T} \cdot\left(\mathbf{R}+\mathbf{H}_{i} \mathbf{P} \mathbf{H}_{i}^{T}\right)^{-1} \cdot\left(\mathbf{y}-\mathbf{H}_{i} \mathbf{x}^{\mathrm{b}}\right)$

and

$\mathbf{A}^{-1}=\mathbf{P}^{-1}+\mathbf{H}_{i}^{T} \mathbf{R}^{-1} \mathbf{H}_{i}$.

Note that Eqs. (A8) and (A9) are the standard expressions for the posterior mean and variance of $\mathbf{x}$. Now substituting in Eq. (A2) and using the fact that

$$
\begin{aligned}
& \int \mathrm{d} x \exp \left[-\frac{1}{2}(\mathbf{x}-\mu)^{T} \cdot \mathbf{A}^{-1} \cdot(\mathbf{x}-\mu)\right] \\
& =|\mathbf{A}|^{1 / 2}(2 \pi)^{n / 2},
\end{aligned}
$$

we have

$$
\begin{gathered}
p\left(\mathbf{H}_{i}\right) \propto\left|\mathbf{A} \mathbf{P}^{-1}\right|\left|\mathbf{R}^{-1}\right| \exp \left[-\frac{1}{2}\left(\mathbf{y}-\mathbf{H}_{i} \mathbf{x}^{\mathbf{b}}\right)^{T}\right. \\
\left..\left(\mathbf{R}+\mathbf{H}_{i} \mathbf{P} \mathbf{H}^{T}\right)^{-1} \cdot\left(\mathbf{y}-\mathbf{H}_{i} \mathbf{x}^{\mathbf{b}}\right)\right] .
\end{gathered}
$$

We also have the condition that

$$
\sum_{i} p\left(\mathbf{H}_{i}\right)=1 \text {. }
$$

Finally we can simplify $\left|\mathbf{A} \mathbf{P}^{-1}\right|\left|\mathbf{R}^{-1}\right|$ using Eq. (A6) and Sylvester's determinant theorem, which states that for any matrices $\mathbf{U}$ and $\mathbf{V}$

$$
|\mathbf{I}+\mathbf{U V}|=|\mathbf{I}+\mathbf{V U}| .
$$

Substituting and simplifying yields

$$
\begin{gathered}
p\left(\mathbf{H}_{i}\right) \propto\left|\mathbf{R}+\mathbf{H}_{i} \mathbf{P} \mathbf{H}_{i}^{\mathrm{T}}\right|^{-1 / 2} \exp \left[-\frac{1}{2}\left(\mathbf{y}-\mathbf{H}_{i} \mathbf{x}^{\mathrm{b}}\right)^{T}\right. \\
\left..\left(\mathbf{R}+\mathbf{H}_{i} \mathbf{P} \mathbf{H}_{i}^{T}\right)^{-1} \cdot\left(\mathbf{y}-\mathbf{H}_{i} \mathbf{x}^{\mathrm{b}}\right)\right] .
\end{gathered}
$$


Competing interests. The author declares that there is no conflict of interest.

Special issue statement. This article is part of the special issue "Data assimilation in carbon/biogeochemical cycles: consistent assimilation of multiple data streams (BG/ACP/GMD inter-journal $\mathrm{SI})$ ". It is not associated with a conference.

Acknowledgements. We acknowledge the support from the International Space Science Institute (ISSI). This publication is an outcome of the ISSI's Working Group "Carbon Cycle Data Assimilation: How to consistently assimilate multiple data streams".

Review statement. This paper was edited by Christoph Gerbig and reviewed by Amy Braverman and two anonymous referees.

Financial support. This research was supported by the Australian Research Council (grant no. DP1096309).

\section{References}

Akaike, H.: A new look at the statistical model identification, IEEE Trans. Auto. Control, 19, 716-723, 1974.

Baker, D. F., Law, R. M., Gurney, K. R., Rayner, P., Peylin, P., Denning, A. S., Bousquet, P., Bruhwiler, L., Chen, Y.-H., Ciais, P., Fung, I. Y., Heimann, M., John, J., Maki, T., Maksyutov, S., Masarie, K., Prather, M., Pak, B., Taguchi, S., and Zhu, Z.: TransCom 3 inversion intercomparison: Impact of transport model errors on the interannual variability of regional $\mathrm{CO}_{2}$ fluxes, 1988-2003, Global Biogeochem. Cy., 20, GB1002, https://doi.org/10.1029/2004GB002439, 2006.

Bodman, R. W., Rayner, P. J., and Karoly, D. J.: Uncertainty in temperature projections reduced using carbon cycle and climate observations, Nat. Clim. Change, 3, 725-729, 2013.

Cressie, N. and Johannesson, G.: Fixed rank kriging for very large spatial data sets, J. Roy. Stat. Soc. B, 70, 209-226, 2008

Cressie, N., Calder, C. A., Clark, J. S., Hoef, J. M. V., and Wikle, C. K.: Accounting for uncertainty in ecological analysis: the strengths and limitations of hierarchical statistical modeling, Ecol. Appl., 19, 553-570, https://doi.org/10.1890/070744.1, 2009.

Desroziers, G., Berre, L., Chapnik, B., and Poli, P.: Diagnosis of observation, background and analysis-error statistics in observation space, Q. J. Roy. Meteorol. Soc., 131, 3385-3396, https://doi.org/10.1256/qj.05.108, 2005.

Fisher, M.: Minimization algorithms for variational data assimilation, in: Proc. ECMWF Seminar on Recent Developments in Numerical Methods for Atmospheric Modelling, 364-385, Reading, 1998.

Friedlingstein, P., Cox, P., Betts, R., Bopp, L., von Bloh, W., Brovkin, V., Doney, S., Eby, M., Fung, I., Govindasamy, B., John, J., Jones, C., Joos, F., Kato, T., Kawamiya, M., Knorr, W., Lindsay, K., Matthews, H. D., Raddatz, T., Rayner, P., Re- ick, C., Roeckner, E., Schnitzler, K.-G., Schnur, R., Strassmann, K., Thompson, S., J. Weaver, A., Yoshikawa, C., and Zeng, N.: Climate-carbon cycle feedback analysis, results from the C4MIP model intercomparison, J. Climate, 19, 3737-3753, https://doi.org/10.1175/JCLI3800.1, 2006.

Ganesan, A. L., Rigby, M., Zammit-Mangion, A., Manning, A. J., Prinn, R. G., Fraser, P. J., Harth, C. M., Kim, K.-R., Krummel, P. B., Li, S., Mühle, J., O’Doherty, S. J., Park, S., Salameh, P. K., Steele, L. P., and Weiss, R. F.: Characterization of uncertainties in atmospheric trace gas inversions using hierarchical Bayesian methods, Atmos. Chem. Phys., 14, 3855-3864, https://doi.org/10.5194/acp-14-3855-2014, 2014.

Griewank, A.: Evaluating Derivatives: Principles and Techniques of Automatic Differentiation, SIAM, Philadelphia, Pa., 2000.

Gurney, K. R., Law, R. M., Denning, A. S., Rayner, P. J., Baker, D., Bousquet, P., Bruhwiler, L., Chen, Y.-H., Ciais, P., Fan, S., Fung, I. Y., Gloor, M., Heimann, M., Higuchi, K., John, J., Maki, T., Maksyutov, S., Masarie, K., Peylin, P., Prather, M., Pak, B. C., Randerson, J., Sarmiento, J., Taguchi, S., Takahashi, T., and Yuen, C.-W.: Towards robust regional estimates of $\mathrm{CO}_{2}$ sources and sinks using atmospheric transport models, Nature, 415, 626630, 2002.

Gurney, K. R., Law, R. M., Denning, A. S., Rayner, P. J., Baker, D., Bousquet, P., Bruhwiler, L., Chen, Y.-H., Ciais, P., Fan, S., Fung, I. Y., Gloor, M., Heimann, M., Higuchi, K., John, J., Kowalczyk, E., Maki, T., Maksyutov, S., Peylin, P., Prather, M., Pak, B. C., Sarmiento, J., Taguchi, S., Takahashi, T., and Yuen, C.-W.: TransCom $3 \mathrm{CO}_{2}$ inversion intercomparison: 1. Annual mean control results and sensitivity to transport and prior flux information, Tellus, 55B, 555-579, https://doi.org/10.1034/j.16000889.2003.00049.x, 2003.

Gurney, K. R., Law, R. M., Denning, A. S., Rayner, P. J., Pak, B. C., Baker, D., Bousquet, P., Bruhwiler, L., Chen, Y.-H., Ciais, P., Fung, I. Y., Heimann, M., John, J., Maki, T., Maksyutov, S., Peylin, P., Prather, M., and Taguchi, S.: Transcom 3 inversion intercomparison: Model mean results for the estimation of seasonal carbon sources and sinks, Global Biogeochem. Cy., 18, GB1010, https://doi.org/10.1029/2003GB002111, 2004.

Heimann, M. and Körner, S.: The global atmospheric tracer model TM3, Tech. Rep. 5, Max Planck Institute for Biogeochemistry, 2003.

Hoeting, J. A., Madigan, D., Raftery, A. E., and Volinsky, C. T.: Bayesian Model Averaging: A Tutorial, Stat. Sci., 14, 382-401, 1999.

Jaynes, E. and Bretthorst, G.: Probability Theory: The Logic of Science, Cambridge University Press, available at: http: //books.google.com.au/books?id=tTN4HuUNXjgC (last access: 28 February 2020), 2003.

Kass, R. E. and Raftery, A. E.: Bayes factors, J. Am. Stat. Assoc., 90, 773-795, 1995.

Lanczos, C.: An iteration method for the solution of the eigenvalue problem of linear differential and integral operators, J. Res. Natl Bur. Stand. B, 45, 255-282, https://doi.org/10.6028/jres.045.026, 1950.

Lauvaux, T., Pannekoucke, O., Sarrat, C., Chevallier, F., Ciais, P., Noilhan, J., and Rayner, P. J.: Structure of the transport uncertainty in mesoscale inversions of $\mathrm{CO}_{2}$ sources and sinks using ensemble model simulations, Biogeosciences, 6, 1089-1102, https://doi.org/10.5194/bg-6-1089-2009, 2009. 
MacKay, D. J. C.: Information Theory, Inference, and Learning Algorithms, Cambridge University Press, available at: http://www. cambridge.org/0521642981 (last access: 28 February 2020) 2003.

Michalak, A. M., Hirsch, A., Bruhwiler, L., Gurney, K. R., Peters, W., and Tans, P. P.: Maximum likelihood estimation of covariance parameters for Bayesian atmospheric trace gas surface flux inversions, J. Geophys. Res., 110, D24107, https://doi.org/10.1029/2005JD005970, 2005.

Murphy, J. M., Booth, B. B., Collins, M., Harris, G. R., Sexton, D. M., and Webb, M. J.: A methodology for probabilistic predictions of regional climate change from perturbed physics ensembles, Philos. Trans. Roy. Soc. A, 365, 1993-2028, 2007.

Peylin, P., Bacour, C., MacBean, N., Leonard, S., Rayner, P., Kuppel, S., Koffi, E., Kane, A., Maignan, F., Chevallier, F., Ciais, P., and Prunet, P.: A new stepwise carbon cycle data assimilation system using multiple data streams to constrain the simulated land surface carbon cycle, Geosci. Model Dev., 9, 3321-3346, https://doi.org/10.5194/gmd-9-3321-2016, 2016.

Pickett-Heaps, C. A., Rayner, P. J., Law, R. M., Bousquet, P., Peylin, P., Patra, P., Maksyutov, S., Marshall, J., Rödenbeck, C., Ciais, P., Langenfelds, R., Tans, P., Steele, P., and Francey, R.: Atmospheric $\mathrm{CO}_{2}$ Inversion Cross-Validation Using Vertical Profile Measurements: Analysis of Four Independent Inversion Models, J. Geophys. Res., 116, D12305, https://doi.org/10.1029/2010JD014887, 2011.

Raftery, A. E., Gneiting, T., Balabdaoui, F., and Polakowski, M.: Using Bayesian Model Averaging to Calibrate Forecast Ensembles, Mon. Weather Rev., 133, 1155-1174, https://doi.org/10.1175/MWR2906.1, 2005.

Rayner, R. J.: Optimizing $\mathrm{CO}_{2}$ observing networks in the presence of model error: results from TransCom 3, Atmos. Chem. Phys., 4, 413-421, https://doi.org/10.5194/acp-4-413-2004, 2004.

Rayner, P. J., Koffi, E., Scholze, M., Kaminski, T., and Dufresne, J.-L.: Constraining predictions of the carbon cycle using data, Phil. Trans. Roy. Soc. A, 369, 1955-1966, https://doi.org/10.1098/rsta.2010.0378, 2011.

Rayner, P.: Code needed to run the transcom ensemble weighted probability case for "Data Assimilation using an Ensemble of Models: A hierarchical approach", Geoscience Model Development Discussions, 2016 w draft item, figshare, https://doi.org/10.4225/49/581cf15db938d, 2016.
Rayner, P. J., Michalak, A. M., and Chevallier, F.: Fundamentals of data assimilation applied to biogeochemistry, Atmos. Chem. Phys., 19, 13911-13932, https://doi.org/10.5194/acp-19-139112019, 2019.

Scholze, M., Kaminski, T., Rayner, P., Knorr, W., and Geiring, R.: Propagating uncertainty through prognostic CCDAS simulations, J. Geophys. Res., 112, d17305, https://doi.org/10.1029/2007JD008642, 2007.

Schwarz, G.: Estimating the Dimension of a Model, Ann. Statist., 6, 461-464, https://doi.org/10.1214/aos/1176344136, 1978.

Stephens, B. B., Gurney, K. R., Tans, P. P., Sweeney, C., Peters, W., Bruhwiler, L., Ciais, P., Ramonet, M., Bousquet, P., Nakazawa, T., Aoki, S., Machida, T., Inoue, G., Vinnichenko, N., Lloyd, J., Jordan, A., Heimann, M., Shibistova, O., Langenfelds, R. L., Steele, L. P., Francey, R. J., and Denning, A. S.: Weak Northern and Strong Tropical Land Carbon Uptake from Vertical Profiles of Atmospheric $\mathrm{CO}_{2}$, Science, 316, 1732-1735, https://doi.org/10.1126/science.1137004, 2007.

Stordal, A. S., Karlsen, H. A., Nævdal, G., Skaug, H. J., and Vallès, B.: Bridging the ensemble Kalman filter and particle filters: the adaptive Gaussian mixture filter, Comput. Geosci., 15, 293-305, 2011.

Takahashi, T., Wanninkhof, R. H., Feely, R. A., Weiss, R. F., Chipman, D. W., Bates, N., Olafsson, J., Sabine, C., and Sutherland, S. C.: Net sea-air $\mathrm{CO}_{2}$ flux over the global oceans: An improved estimate based on the sea-air $p \mathrm{CO}_{2}$ difference, in: Extended abstracts of the 2nd International $\mathrm{CO}_{2}$ in the Oceans Symposium, edited by: Nojiri, Y., 9-15, National Institute for Environmental Studies, 1999.

Tarantola, A.: Inverse Problem Theory: Methods for Data Fitting and Parameter Estimation, Elsevier, Amsterdam, 1987.

Tarantola, A.: Inverse problem theory and methods for model parameter estimation, no. 89 in Other Titles in Applied Mathematics, Society for Industrial and Applied Mathematics, 2005.

Taylor, K. E., Stouffer, R. J., and Meehl, G. A.: An overview of CMIP5 and the experiment design, B. Am. Meteorol. Soc., 93, 485, 2012. 